\title{
Note: Stacked rings for terahertz wave-guiding
}

\author{
E. de Rijk, ${ }^{1,2}$ A. Macor, ${ }^{1,2}$ J-Ph. Hogge, ${ }^{3}$ S. Alberti, ${ }^{3}$ and J-Ph. Ansermet ${ }^{1}$ \\ ${ }^{1}$ Institute of Condensed Matter Physics, Station 3, EPFL, 1015 Lausanne, Switzerland \\ ${ }^{2}$ SWISS to 12 Sàrl, 1015 Lausanne, Switzerland \\ ${ }^{3}$ Centre de Recherche en Physique des Plasmas, Station 13, EPFL, 1015 Lausanne, Switzerland
}

(Received 18 April 2011; accepted 16 May 2011; published online 8 June 2011)

\begin{abstract}
We demonstrate the construction of corrugated waveguides using stacked rings to propagate terahertz frequencies. The waveguide allows propagation of the same fundamental mode as an optical-fiber, namely, the $H E_{11}$ mode. This simple concept opens the way for corrugated wave-guides up to several terahertz, maintaining beam characteristics as for terahertz applications. () 2011 American Institute of Physics. [doi:10.1063/1.3597579]
\end{abstract}

The terahertz frequency range of the electromagnetic spectrum is a frontier area for research in physics, ${ }^{1}$ astrophysics, ${ }^{2} \quad$ plasma-physics, ${ }^{3} \quad$ chemistry, ${ }^{4}$ material science, ${ }^{5}$ biology, ${ }^{6}$ and medicine. ${ }^{7}$ While the "terahertz gap" is starting to be filled with sources, ${ }^{8}$ and detectors ${ }^{9}$ able to generate, and map these THz-fields, ${ }^{10}$ wave-guiding still represents a major issue. ${ }^{11}$ Even if new concepts have been recently proposed, ${ }^{12}$ achieving an ideal transmission line over distances orders of magnitude bigger than the propagated wavelength with a good power coupling to the source, low loss, and low dispersion remains challenging.

Cylindrical corrugated waveguides are widely used to transmit frequencies of several tens of GHz. ${ }^{3,13}$ These waveguides propagate hybrid electric (HE) modes, for which the lowest order $H E_{11}$ mode, as in fiber optics, ${ }^{14}$ has a power loss of the order of $1 \%$ per $100 \mathrm{~m}$, a low dispersion over bandwidth that can reach more than one octave, intrinsic filtering of spurious modes, and an efficient coupling $(\approx 98 \%)$ to the $\mathrm{TEM}_{00}$ "gaussian mode," which is the lowest order mode for free space propagation. ${ }^{3,15}$

The corrugation geometry, Fig. 1(a), depends on the propagated wavelength $\lambda$ and has dimensions and periodicity smaller than $\lambda / 2 .{ }^{3,15,16} \mathrm{Up}$ to now, the corrugation is achieved by conventional machining inside hollow tubes and reaches the current technological limits at several hundreds of $\mathrm{GHz}$, over lengths of few $\mathrm{cm}$ and inner radii on the order of $1 \mathrm{~cm}$. Beyond this limit, the period of the corrugations can no longer be lowered without seriously deteriorating the transmission performances.

In this note we introduce the concept of stacked rings to manufacture $H E_{11}$ cylindrical corrugated waveguides with high mechanical accuracy that meet the geometrical requirements to propagate frequencies up to at least $7.5 \mathrm{THz}$ over modules of tens of centimeters without mechanical limitations on the inner radii.

Corrugations are built by alternately stacking two sets of metallic rings having the same external shape but different inner diameters and thickness inside a guiding pipe, Fig. 1. Rings are obtained from high precision laminated stainless steel sheets commonly available with thickness down to 10 $\pm 1 \mu \mathrm{m}$, cut by electric discharge machining, guaranteeing a cut accuracy of $\pm 2 \mu \mathrm{m}$.
Based on the idea of stacked rings, two $40 \mathrm{~cm}$ long sections of $H E_{11}$ corrugated waveguides were assembled, Fig. 2(a). By using 4000 rings with a thickness of 0.1 and $0.3 \mathrm{~mm}(\mathrm{~d}=0.3 \mathrm{~mm}, \mathrm{D}=19.3 \mathrm{~mm})$, a corrugation that fits the propagation of the $H E_{11}$ mode with a nominal frequency of $0.26 \mathrm{THz}$ is achieved. This frequency corresponds to the electron resonance in state-of-the-art dynamic nuclear polarization enhanced nuclear magnetic resonance experiments ${ }^{4}$ ongoing at the EPFL, which motivated this work.

The robustness of the method has been tested by the realization of a $10 \mathrm{~cm}$ long section designed for the propagation of the $H E_{11}$ mode at $1.5 \mathrm{THz}(\mathrm{p}=0.1 \mathrm{~mm}, \mathrm{~d}=0.05, \mathrm{w}=0.05$, $\mathrm{D}=19.3$ ). The prototypes have been built with auto-aligning connection flanges allowing a modular design, a vacuum compatibility for possible high power applications, and by ensuring the continuity of the corrugation between two segments. Furthermore, the concept of stacked rings can eventually be used to manufacture complex corrugated antennas and horns.

Measurements of the waveguide transmission performances were achieved by using a $14 \mathrm{~mW} \quad 0.25-0.27 \mathrm{THz}$ tunable solid-state source producing a quasi-gaussian linearly polarized beam in conjunction with a Schottky detection diode mounted on a 3D translational stage to map the intensity of the oscillating terahertz field, Fig. 2(b). A dielectric

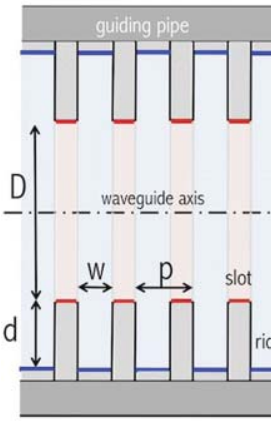

(a)

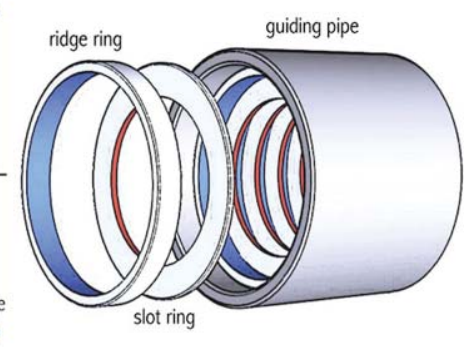

(b)
FIG. 1. (Color online) Corrugation from stacked rings. (a) A schematic view of an $H E_{11}$ corrugated waveguide. For the desired $\mathrm{HE}_{11}$ mode to be sustained, the following characteristics need to be met: $\mathrm{p}<\lambda / 2, \mathrm{~d} \approx \lambda / 4$, w as large as possible and $\mathrm{D} \gg \lambda^{3,15,16}$ (b) The corrugation is obtained by alternately piling up slot and ridge rings into the guiding pipe. 


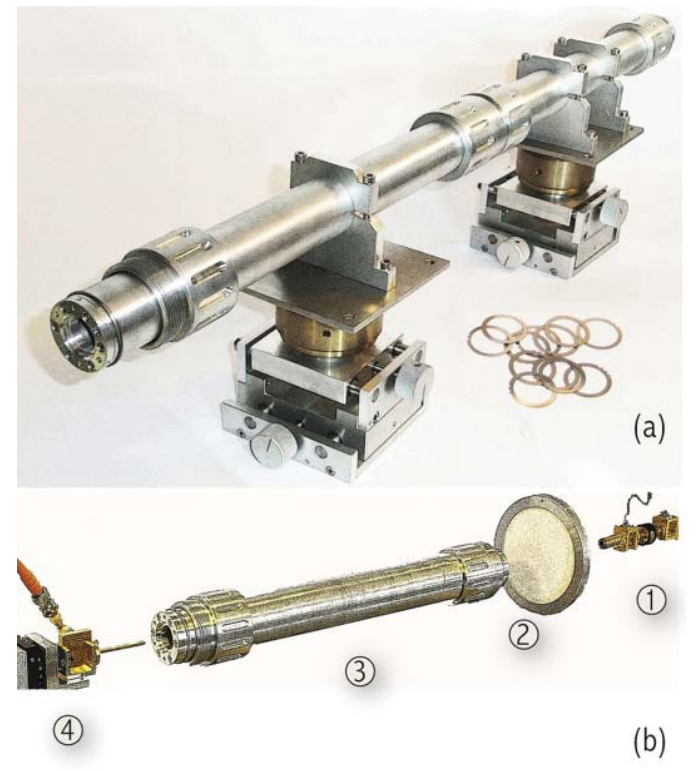

FIG. 2. (Color online) Waveguide prototype testing. (a) Two connected $H E_{11}$ corrugated waveguide modules, $80 \mathrm{~cm}$ long, mounted on positioning stages. The guiding pipe is made of aluminum and the stacked rings inside are of stainless steel. Rings can be seen next to the waveguide. (b) The experimental setup with: (1) the source output horn producing a quasi-gaussian beam at $0.26 \mathrm{THz},(2)$ the dielectric lens, (3) the $H E_{11}$ corrugated waveguide, (4) Schottky diode used for detection with tangential signal sensitivity (T.S.S.) of $0.04 \mu \mathrm{W}$, connected to a small fundamental rectangular waveguide for signal collection, sensitive to the power flow of the linearly polarized beam over a surface of $0.4 \mathrm{~mm} \times 0.8 \mathrm{~mm}$.

lens was used to reshape the beam produced by the source so as to optimize the coupling with the waveguide and excite a high purity $H E_{11}$ mode. This matching condition is reached when a gaussian beam is injected with plane phase front at the waveguide aperature with a beam spot size, $w_{0}$, satisfying $2 w_{0} / D=0.64 .{ }^{16}$ The corrugated waveguide being an overmoded environment with respect to the injected wavelength, bad coupling conditions can excite higher order HE modes. Although these modes are damped significantly faster than the $H E_{11}$ mode, they can still be sustained over several meters. ${ }^{17}$

Measured terahertz beam intensity profiles over several cross sections enable the use of phase reconstruction algorithms to determine the modal expansion of the beam. ${ }^{18}$ The phase reconstruction algorithm is an iterative calculation that uses a free space propagator to retrieve the phase information from intensity measurements only. We used this technique to reconstruct the electric field amplitude and phase profile of the beam injected into the transmission line, Fig. 3, after propagating through one $40 \mathrm{~cm}$ long module and after propagating through two connected waveguide modules with a total length of $80 \mathrm{~cm}$, Fig. 4 .

When performing a modal expansion on the reconstructed information of the beam injected into the waveguide we identified a $94.9 \pm 0.1 \%$ gaussian content. In theory with the given waveguide features, the gaussian mode propagating in free space couples to the $H E_{11}$ mode, and vice versa, with an efficiency of about $98.1 \%$, mostly due to the truncation introduced by the waveguide aperture. ${ }^{19}$ In our case, the coupling from the source's beam to the waveguide was found to be $96.7 \pm 0.1 \%$.

The modal expansion performed on the beam radiated after $40 \mathrm{~cm}$ of propagation through the waveguide yielded a $H E_{11}$ content of $96.4 \pm 0.1 \%$ on the output aperture. Finally after $80 \mathrm{~cm}$ of propagation through the waveguide the $H E_{11}$ content was of $98.1 \pm 0.1 \%$.

This analysis confirms that the waveguide acts as a modal filter where higher order modes are damped and the $H E_{11}$ mode experiences a low power loss theoretically predicted to be of the order of $1 \%$ per $100 \mathrm{~m}$.

The comparison of the raw experimental beam profiles after a $40 \mathrm{~cm}$ section of waveguide and after two connected sections $(80 \mathrm{~cm})$ does not show any measurable power loss above the $2 \%$ precision of the measurements mostly due to thermal drifts of source's output power.

The experimental results have therefore demonstrated the robustness of the presented method to manufacture $H E_{11}$ corrugated waveguides extending their range of applicability from the field of microwaves to the domain of terahertz waves. A $96.7 \pm 0.1 \%$ power coupling to the waveguide has been obtained as well as a low propagation loss owing to the high purity $H E_{11}$ mode $(98.1 \pm 0.1 \%$ after $80 \mathrm{~cm})$, propagating inside the waveguide at $0.26 \mathrm{THz}$. The mechanical feasibility of the waveguide for frequencies up to $7.5 \mathrm{THz}$ stems from

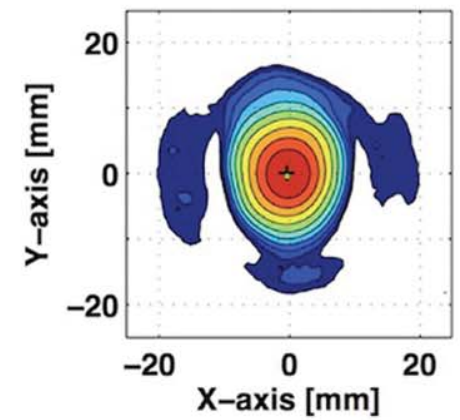

(a)

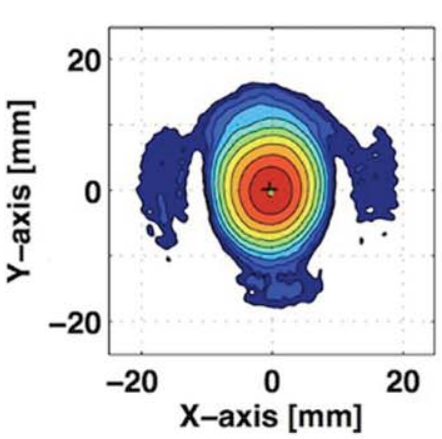

(b)
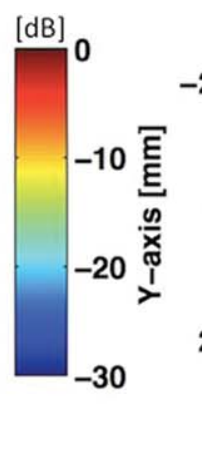

(c)

FIG. 3. (Color online) Phase retrieval of the source. Measured and numerically reconstructed data of the quasi-gaussian beam at $0.26 \mathrm{THz}$ injected into the aperture of the waveguide. (a) Normalized measured intensity profile corresponding to the cross section of the beam injected into the waveguide. The sampling step is of $0.5 \mathrm{~mm}$ on both axes. (b) Intensity profile resulting from the phase reconstruction on the cross section of the beam injected into the waveguide, after a convergence $>99.9 \%$. (c) Computed phase distribution profile of the electric field resulting from the phase reconstruction at the cross section shown in (b). 


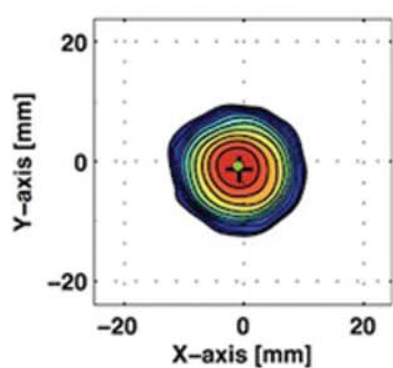

(a)

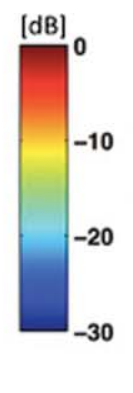

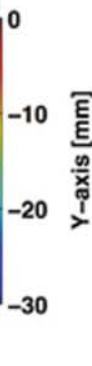

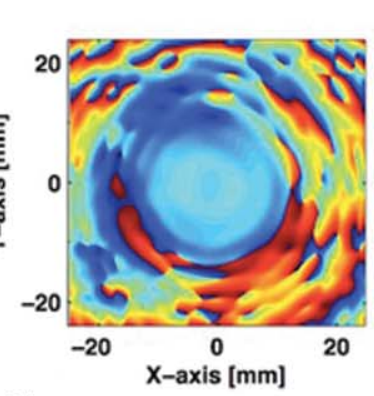

(b)

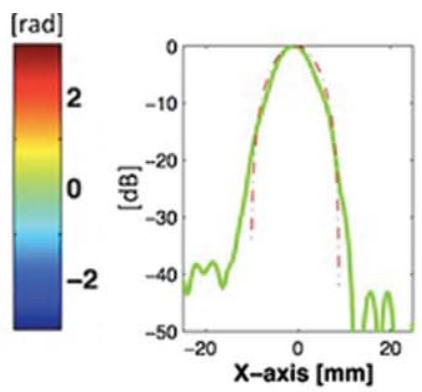

(c)

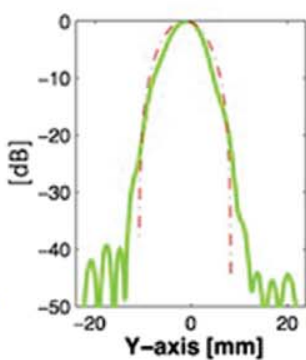

(d)

FIG. 4. (Color online) Purity of the propagated $\mathrm{HE}_{11}$. (a) Reconstructed intensity profile at $0.26 \mathrm{THz}$ at the aperture after two consecutive modules of waveguide $(80 \mathrm{~cm})$, obtained by using three different measured intensity profiles to run the phase reconstruction algorithm with a convergence $>99.9 \%$. (b) Reconstructed phase profile of the electric field at $0.26 \mathrm{THz}$ at the aperture after two consecutive modules. (c) Section along the X-axis of (a) corresponding to the direction perpendicular to the E field polarization (Fig. 2), passing through the center of gravity of the beam (green solid lines), along with the profile of the best fitting $H E_{11}$ mode distribution (red dots). (d) Section along the Y-axis (parallel to the E-field polarization) of (a) passing through the center of gravity of the beam (green solid lines), along with the profile of the best fitting $H E_{11}$ mode distribution (red dashed line).

the availability of sheet metal of thickness as low as 10 microns, and state-of-the-art electric discharge machining.

Work supported by Requip (No. 206021-121303/1), Sinergia (No. CRSI20-122708/1), and (No. 200020-120503/1) grants of the Swiss National Science Foundation and by the Ecole Polytechnique Fédérale de Lausanne.

The authors thank the technical staff of the ICMP and the CRPP, especially G. Grandjean, P. Zuercher, S. Allenspach, R. Chavan, and R. Bertizzolo for their contribution to this work.

${ }^{1}$ B. E. Cole, J. B. Williams, B. T. King, M. S. Sherwin, and C. R. Stanley, Nature (London) 410, 60 (2001).

${ }^{2}$ G. L. Pilbratt, J. R. Riedinger, T. Passvogel, G. Crone, D. Doyle, U. Gageur, A. M. Heras, C. Jewell, L. Metcalfe, S. Ott, and M. Schmidt, Astron. Astrophys. 518, L1 (2010).

${ }^{3}$ J. L. Doane, Fusion Sci. Technol. 53, 159 (2008).

${ }^{4}$ R. G. Griffin and T. F. Prisner, Phys. Chem. Chem. Phys. 12, 5737 (2010).
${ }^{5}$ B. Ferguson and X. C. Zhang, Nature Mater. 1, 26 (2002).

${ }^{6}$ X. C. Zhang, Nat. Photonics 4, 662 (2010).

${ }^{7}$ M. B. Johnston, Nat. Photonics 1, 14 (2007).

${ }^{8}$ S. Kumar, C. W. I. Chan, Q. Hu, and J. L. Reno, Nat. Phys. 7, 166 (2011).

${ }^{9}$ W. Knap, M. Dyakonov, D. Coquillat, F. Teppe, N. Dyakonova, J. Lusakowski, K. Karpierz, M. Sakowicz, G. Valusis, D. Seliuta, I. Kasalynas, A. El Fatimy, Y. M. Meziani and T. Otsuji, J. Infrared Milli. Terahz. Waves 30, 1319 (2009).

${ }^{10}$ M. Tonouchi, Nat. Photonics 1, 97 (2007).

${ }^{11}$ M. Sherwin, C. A. Schmuttenmaer, and P. H. E. Bucksbaum, Report on DOE-NSF-NIH Workshop, 65, 76-77, 100-101 (2004).

${ }^{12}$ K. Wang and D. M. Mittleman, Nat. Photonics 432, 376 (2004).

${ }^{13}$ G. R. Hanson, J. B. Wilgen, T. S. Bigelow, S. J. Diem, and T. M. Biewer, Rev. Sci. Instrum. 81, 10D920 (2010).

${ }^{14}$ C. K. Kao, Optical Fibre Technology II (IEEE Press, 1981).

${ }^{15} \mathrm{P}$. Clarricoats, Corrugated horns for microwave antennas, IEE Electromagnetic waves series, $\mathbf{1 8}, 1984$.

${ }^{16}$ J. P. Crenn, Int. J. Infrared Millim. Waves 14, 1947 (1993).

${ }^{17}$ H. Idei, T. Shimozuma, M. A. Shapiro, T. Notake, S. Kubo, and R. J. Temkin, IEEE Trans. Microwave Theory Tech 54, 3899 (2006).

${ }^{18}$ S. Jawla, EPFL Ph.D. thesis, No. 4675, 2010.

${ }^{19}$ M. Shapiro and R. J. Temkin, J. Infrared Millim. Terahz. Waves 32, 283 (2011). 